\title{
Action Principle for Newtonian Gravity
}

\author{
Dennis Hansen, ${ }^{1, *}$ Jelle Hartong, ${ }^{2, \dagger}$ and Niels A. Obers ${ }^{3, \$}$ \\ ${ }^{1}$ Institut für Theoretische Physik, Eidgenössische Technische Hochschule Zürich Wolfgang-Pauli-Strasse 27, 8093 Zürich, Switzerland \\ ${ }^{2}$ School of Mathematics and Maxwell Institute for Mathematical Sciences, University of Edinburgh, \\ Peter Guthrie Tait Road, Edinburgh EH9 3FD, United Kingdom \\ ${ }^{3}$ The Niels Bohr Institute, Copenhagen University, Blegdamsvej 17, DK-2100 Copenhagen Ø, Denmark
}

(Received 9 October 2018; published 15 February 2019)

\begin{abstract}
We derive an action whose equations of motion contain the Poisson equation of Newtonian gravity. The construction requires a new notion of Newton-Cartan geometry based on an underlying symmetry algebra that differs from the usual Bargmann algebra. This geometry naturally arises in a covariant $1 / c$ expansion of general relativity, with $c$ being the speed of light. By truncating this expansion at subleading order, we obtain the field content and transformation rules of the fields that appear in the action of Newtonian gravity. The equations of motion generalize Newtonian gravity by allowing for the effect of gravitational time dilation due to strong gravitational fields.
\end{abstract}

DOI: 10.1103/PhysRevLett.122.061106

The idea that gravity is geometry was pioneered by Einstein in his celebrated theory of general relativity (GR). In GR, due to Einstein's equivalence principle, the underlying geometry is (pseudo-)Riemannian, which ensures that one has local Lorentz symmetry and hence the laws of physics locally reduce to those of special relativity. However, spacetime covariance is a property of any physical theory, which led Cartan [1,2] (see also, e.g., $[3,4]$ ) to geometrize Newtonian gravity using what is known as Newton-Cartan (NC) geometry. The latter results from applying an equivalence principle that requires freely falling observers to see Galilean laws of physics, giving rise to a geometry with local Galilean invariance.

However, while the Poisson equation of Newtonian gravity can be geometrized using NC geometry, an outstanding question has been to find an action principle for Newtonian gravity, paralleling the Einstein-Hilbert action in GR. In this Letter we present such an action and show that it requires a novel type of geometry. This geometry does encapsulate NC geometry in its original form when time is absolute (as is the case in Newtonian gravity), but is based on an underlying symmetry structure and corresponding set of geometric fields, which goes beyond the Bargmann algebra - the centrally extended Galilean algebra.

$\mathrm{NC}$ geometry and its recently discovered version, torsional Newton-Cartan (TNC) geometry [5,6] (referred to as type I TNC geometry below), has been very useful for studying

Published by the American Physical Society under the terms of the Creative Commons Attribution 4.0 International license. Further distribution of this work must maintain attribution to the author(s) and the published article's title, journal citation, and DOI. Funded by SCOAP ${ }^{3}$. aspects of field theories with Galilean symmetries. Furthermore, gravity theories for type I TNC geometry (with broken particle number gauge symmetry) have been recently studied as well and shown to correspond to Hořava-Lifshitz gravity (see, e.g., $[7,8])$. It has proven difficult to write down actions for type I TNC geometry that preserve U(1) particle number, though exceptions exist in $2+1$ dimensions $[9,10]$, but these require an additional field.

By taking a critical look at Newtonian gravity, we will show that an action involving type I TNC geometry is incompatible with the way in which the mass source appears in the Poisson equation. This is because in type I TNC geometry mass sources torsion, which is not compatible with the notion of absolute time (and hence zero torsion) of Newtonian gravity. The key to identifying the correct geometry lies in carefully considering the properties of a large speed of light limit of GR, as was recently revisited in [11] following earlier work $[12,13]$.

We present in this Letter a novel type of NC geometry, dubbed type II TNC geometry, which for zero torsion includes the standard (type I) NC geometry used to geometrize Newtonian gravity, and which allows us to formulate an action, in any spacetime dimension $D=d+1$. To this end, it is crucial to allow for more general time (lapse) functions than the absolute time of Newtonian gravity. We will show that, while type I TNC geometry follows from gauging the Bargmann algebra $[7,14]$ (see also $[15,16]$ ), type II TNC geometry follows from a novel nonrelativistic symmetry, which turns out to be a nontrivial contraction of the direct sum of the Poincare and Euclidean algebras in $D=d+1$ dimensions.

The action given in this Letter describes the dynamics of a well-defined truncation of the nonrelativistic limit of GR and has direct physical relevance in a post-Newtonian 
regime, including the effects of strong gravitational fields, e.g., via gravitational time dilation. More generally, it can be regarded as providing an off shell definition of the nonrelativistic gravity corner of the $G c \hbar$ Bronstein cube of physical theories and, as such, presents a principle towards constructing a nonrelativistic quantum gravity theory. The latter could open up a third road towards (relativistic) quantum gravity, in contradistinction to the usually traveled paths approaching it via relativistic quantum field theory or general relativity.

Newton-Cartan geometry.-Torsional Newton-Cartan geometry consists of a clock form $\tau_{\mu}$, a rank- $d$ symmetric tensor $h_{\mu \nu}$ with signature $(0,1, \ldots, 1)$, and a U(1) connection $m_{\mu}$. These describe a manifold with a Galilean tangent space structure, geometrizing the Galilean equivalence principle. In Galilean invariant theories, the total mass is conserved with the mass current coupling to a $\mathrm{U}(1)$ gauge connection $m_{\mu}$. The TNC fields transform as tensors under diffeomorphisms $\left(\xi^{\mu}\right)$ and exhibit furthermore a set of local symmetries corresponding to Galilean (or Milne) boosts $\left(\lambda_{\mu}\right)$ and a $\mathrm{U}(1)$ gauge transformation $(\sigma)$ associated with mass conservation,

$$
\begin{aligned}
\delta \tau_{\mu} & =\mathcal{L}_{\xi} \tau_{\mu}, \quad \delta h_{\mu \nu}=\mathcal{L}_{\xi} h_{\mu \nu}+\lambda_{\mu} \tau_{\nu}+\lambda_{\nu} \tau_{\mu}, \\
\delta m_{\mu} & =\mathcal{L}_{\xi} m_{\mu}+\lambda_{\mu}+\partial_{\mu} \sigma,
\end{aligned}
$$

where $\mathcal{L}_{\xi}$ denotes the Lie derivative along $\xi^{\mu}$. The geometric tensors $v^{\mu}$ and $h^{\mu \nu}$ are defined by inverting $-\tau_{\mu} \tau_{\nu}+h_{\mu \nu}$ to $-v^{\mu} v^{\nu}+h^{\mu \nu}$, with the property that $\tau_{\mu} h^{\mu \nu}=0$ and $v^{\mu} \tau_{\mu}=-1$. The Galilean boost parameters satisfy $v^{\mu} \lambda_{\mu}=0$. The analog of the absolute value of the determinant of the pseudo-Riemannian metric, which for TNC we denote by $e^{2}$, is given by minus the determinant of the matrix $-\tau_{\mu} \tau_{\nu}+h_{\mu \nu}$. Three useful tensors that are invariant under local Galilean boosts (and rotations) are $\hat{v}^{\mu} \equiv v^{\mu}-h^{\mu \nu} m_{\nu}$, $\bar{h}_{\mu \nu} \equiv h_{\mu \nu}-2 \tau_{(\mu} m_{\nu)}$, and $\tilde{\Phi} \equiv-v^{\mu} m_{\mu}+\frac{1}{2} h^{\mu \nu} m_{\mu} m_{\nu}$. We also record the completeness relation $-\hat{v}^{\mu} \tau_{\nu}+h^{\mu \lambda} \bar{h}_{\lambda \nu}=\delta_{\nu}^{\mu}$.

We will choose the following affine connection to perform covariant differentiation [17-20]:

$$
\bar{\Gamma}_{\mu \nu}^{\lambda} \equiv-\hat{v}^{\lambda} \partial_{\mu} \tau_{\nu}+\frac{1}{2} h^{\lambda \sigma}\left(\partial_{\mu} \bar{h}_{\nu \sigma}+\partial_{\nu} \bar{h}_{\mu \sigma}-\partial_{\sigma} \bar{h}_{\mu \nu}\right) .
$$

This is a metric compatible connection, i.e., $\bar{\nabla}_{\mu} \tau_{\nu}=0=$ $\bar{\nabla}_{\mu} h^{\nu \rho}$. Note that this connection is not invariant under the local $\mathrm{U}(1)$ transformation with parameter $\sigma$. In TNC geometry we cannot make the local Galilean boost and local U(1) symmetries manifest at the same time. We also note that this connection has torsion because $\bar{\Gamma}_{[\mu \nu]}^{\lambda}=-\hat{v}^{\lambda} \partial_{[\mu} \tau_{\nu]}$. When the clock 1-form $\tau_{\mu}$ obeys $h^{\mu \rho} h^{\nu \sigma}\left(\partial_{\mu} \tau_{\nu}-\partial_{\nu} \tau_{\mu}\right)=0$, we call the torsion twistless and the resulting geometry is called twistless torsional Newton-Cartan (TTNC) geometry [5-7]. In this Letter we will assume throughout that $\tau_{\mu}$ is twistless, implying that $\tau_{\mu}$ obeys the Frobenius integrability condition $\tau_{[\mu} \partial_{\nu} \tau_{\rho]}=0$ so that $\tau_{\mu}$ is hypersurface orthogonal. Thus, in this case, the spacetime allows a foliation in terms of equal-time slices.

A useful property of the connection (2) is $\bar{\Gamma}_{\rho \mu}^{\rho}=$ $e^{-1} \partial_{\mu} e-a_{\mu}$, where we defined the torsion vector $a_{\mu} \equiv$ $\mathcal{L}_{\hat{v}} \tau_{\mu}$ and $e$ has been defined above. This implies $\left(\bar{\nabla}_{\mu}+a_{\mu}\right) X^{\mu}=e^{-1} \partial_{\mu}\left(e X^{\mu}\right)$. We define the associated Riemann tensor as usual via

$$
\left[\bar{\nabla}_{\mu}, \bar{\nabla}_{\nu}\right] X_{\sigma}=\bar{R}_{\mu \nu \sigma}^{\rho} X_{\rho}-2 \bar{\Gamma}_{[\mu \nu]}^{\rho} \bar{\nabla}_{\rho} X_{\sigma} .
$$

Further, we define the Ricci tensor as $\bar{R}_{\mu \nu}=\bar{R}_{\mu \rho \nu}^{\rho}$. Because of the presence of torsion, one can show using the Bianchi identity for $\bar{R}_{[\mu \nu \sigma]}{ }^{\rho}$ that the antisymmetric part of the Ricci tensor is nonzero and equal to

$2 \bar{R}_{[\mu \nu]}=\left(\tau_{\mu} a_{\nu}-\tau_{\nu} a_{\mu}\right) \bar{\nabla}_{\rho} \hat{v}^{\rho}+\hat{v}^{\rho}\left(\tau_{\mu} \bar{\nabla}_{\nu} a_{\rho}-\tau_{\nu} \bar{\nabla}_{\mu} a_{\rho}\right)$.

The above reviewed standard TNC geometry is referred to as type I TNC geometry below.

Finally, we note that a convenient way to think of type I TNC geometry is via the process of null uplift [21], which will be instrumental below in showing that this geometry cannot correctly describe Newtonian gravity. Any TNC geometry can be written as a Lorentzian geometry with a null isometry in one dimension higher. Parametrizing the null isometry with $u$ we can write the Lorentzian metric $\hat{g}_{M N}$ as

$\hat{g}_{M N} d x^{M} d x^{N}=2 \tau_{\mu} d x^{\mu}\left(d u-m_{\nu} d x^{\nu}\right)+h_{\mu \nu} d x^{\mu} d x^{\nu}$,

where $x^{M}=\left(u, x^{\mu}\right)$. The null Killing vector is $\partial_{u}$. The inverse metric is $\hat{g}^{u u}=2 \tilde{\Phi}, \hat{g}^{\mu u}=-\hat{v}^{\mu}$, and $\hat{g}^{\mu \nu}=h^{\mu \nu}$. At the level of symmetries, the null reduction means that the Bargmann algebra is a subalgebra of Poincaré in one dimension higher. Alternatively, it can be obtained by an Inönü-Wigner contraction of the product of the Poincare algebra (in the same dimension) times a U(1).

A critical look at Newton-Cartan gravity.-Type I Newton-Cartan geometry was initially invented to describe Newtonian gravity in a coordinate independent manner. The equations of motion that covariantize the Poisson equation of Newtonian gravity are

$$
\bar{R}_{\mu \nu}=8 \pi G \frac{d-2}{d-1} \rho \tau_{\mu} \tau_{\nu}, \quad \partial_{\mu} \tau_{\nu}-\partial_{\nu} \tau_{\mu}=0,
$$

where $\rho$ is the mass density. On flat spacetime in Cartesian coordinates $\tau=d t$ and $h_{\mu \nu} d x^{\mu} d x^{\nu}=d x^{i} d x^{i}$ with $m=$ $\Phi d t$, this simply reduces to

$$
\partial_{i} \partial_{i} \Phi=8 \pi G \frac{d-2}{d-1} \rho .
$$


Both sides of (6) are formulated in terms of NC objects and are invariant under all type I NC gauge symmetries for $d \tau=0$. Yet, the coupling of mass to the geometry is not what one would expect from a theory with local Bargmann $\mathrm{U}(1)$ invariance. The gauge field $m_{\mu}$ couples to the conserved mass current $J^{\mu}$, so any type I invariant action leads to an equation of the form $R^{\mu}=J^{\mu}$, where the lefthand side is a geometrical object formed from the type I TNC fields obeying the Bianchi identity $\partial_{\mu}\left(e R^{\mu}\right)=0$.

Using null uplift (5), the equation $R^{\mu}=J^{\mu}$ reads $\hat{G}_{u}^{\mu}=8 \pi G \hat{T}_{u}^{\mu}$, where $\hat{G}_{N}^{M}$ and $\hat{T}_{N}^{M}$ are the higher dimensional Einstein and energy-momentum tensors. From the Bianchi identity for $\hat{G}_{N}^{M}$, it follows that $\hat{G}_{u}^{\mu}$ is identically conserved. Contracting with $\tau_{\mu}$ we see that mass sources $\tau \wedge d \tau \neq 0$ since $\hat{G}_{u u}=8 \pi G \hat{T}_{u u} \equiv 8 \pi G \rho$ with $\hat{G}_{u u}=$ $\frac{1}{4}\left[h^{\mu \rho} h^{\nu \sigma}\left(\partial_{\mu} \tau_{\nu}-\partial_{\nu} \tau_{\mu}\right)\left(\partial_{\rho} \tau_{\sigma}-\partial_{\sigma} \tau_{\rho}\right)\right]^{2}$. This conflicts with Newtonian gravity since in that case the notion of mass is compatible with $d \tau=0$; i.e., $\rho$ in Newton's law is not a Bargmann mass.

Newtonian gravity is obtained from a nonrelativistic limit of GR, but we have just shown that this limit cannot be type I TNC geometry. This begs the question what kind of geometry one should employ. The answer comes from studying the large speed of light limit of GR, i.e., the $1 / c$ expansion of $[11,12]$. We will show that this leads to a different notion of Newton-Cartan geometry, which we call type II Newton-Cartan geometry, and that this allows for an off shell formulation of Newtonian gravity.

$1 / c$ expansion and type II TNC geometry.-In a $1 / c$ expansion the pseudo-Riemannian metric and its inverse are expanded as [11]

$$
g_{\mu \nu}=-c^{2} \tau_{\mu} \tau_{\nu}+\bar{h}_{\mu \nu}+\frac{1}{c^{2}}\left(2 \tau_{(\mu} \hat{B}_{\nu)}-\bar{h}_{\mu \rho} \bar{h}_{\nu \sigma} \hat{\beta}^{\rho \sigma}\right)+\mathcal{O}\left(c^{-4}\right),
$$

$$
g^{\mu \nu}=h^{\mu \nu}-\frac{1}{c^{2}} \hat{v}^{\mu} \hat{v}^{\nu}+\frac{1}{c^{2}} \hat{\beta}^{\mu \nu}+\mathcal{O}\left(c^{-4}\right),
$$

where we note that the 1 -form $\hat{B}_{\mu}$ will play no role in what follows. It is convenient to define $\Phi_{\mu \nu}$ via the relation $\hat{\beta}^{\mu \nu}=h^{\mu \rho} h^{\nu \sigma} \Phi_{\rho \sigma}$.

Using the corresponding $1 / c$ expansion for the vielbeins [22], it follows that the fields $\tau_{\mu}$ and $h_{\mu \nu}$ appearing above transform as in type I TNC geometry [see (1)]. In addition, the fields $m_{\mu}$ and $\Phi_{\mu \nu}$ transform as

$$
\begin{aligned}
\delta m_{\mu} & =\mathcal{L}_{\xi} m_{\mu}+\lambda_{\mu}+\left(\partial_{\mu}-a_{\mu}\right) \lambda+\tau_{\mu} h^{\rho \nu} a_{\rho} \zeta_{\nu} \\
\delta \Phi_{\mu \nu} & =\mathcal{L}_{\xi} \Phi_{\mu \nu}-2 \lambda \bar{K}_{\mu \nu}+\bar{\nabla}_{\mu} \zeta_{\nu}+\bar{\nabla}_{\nu} \zeta_{\mu},
\end{aligned}
$$

where we defined $\lambda$ and $\zeta_{\nu}$ through the equation $\zeta^{\mu}=$ $-\hat{v}^{\mu} \lambda+h^{\mu \nu} \zeta_{\nu}, \bar{K}_{\mu \nu} \equiv-\frac{1}{2} \mathcal{L}_{\hat{v}} \bar{h}_{\mu \nu}$ is the extrinsic curvature tensor, and we recall that $a_{\mu} \equiv \mathcal{L}_{\hat{v}} \tau_{\mu}$ is the torsion vector.
These important extra symmetries follow from expanding relativistic diffeomorphism $\Xi^{\mu}=\xi^{\mu}+\left(1 / c^{2}\right) \zeta^{\mu}+\cdots$, so that $\xi^{\mu}$ parametrizes nonrelativistic diffeomorphisms and $\zeta^{\mu}$ the extra symmetries above.

We will refer to the $\lambda$ transformation in (10) as a torsional $\mathrm{U}(1)$ transformation due to the presence of the torsion vector $a_{\mu}$. One notices that for $d \tau=0$ the transformation of $m_{\mu}$ above reduces to the one in (1), since the $\zeta_{\nu}$ part vanishes in that case while the torsional $U(1)$ takes the same form as the U(1) transformation in (1). However, the gauge field $m_{\mu}$ in type II TNC geometry is quite different from its type I cousin. In particular, we will show in [22] that in type II TNC geometry $m_{\mu}$ couples to the energy current as opposed to type I where it couples to the mass current.

Deferring details to [22] we remark that the transformations of the type II TNC geometry introduced above can be obtained by gauging a novel nonrelativistic algebra of dimension $(d+1)(d+2)$, spanned by the generators $\left\{H, P_{a}, G_{a}, J_{a b}\right\}$ of the (massless) Galilean algebra augmented with the set $\left\{N, T_{a}, B_{a}, S_{a b}\right\}$, with nonzero commutators

$$
\begin{aligned}
{\left[H, G_{a}\right] } & =P_{a}, \quad\left[P_{a}, G_{b}\right]=N \delta_{a b}, \quad\left[N, G_{a}\right]=T_{a}, \\
{\left[H, B_{a}\right] } & =T_{a}, \quad\left[S_{a b}, P_{c}\right]=2 \delta_{c[a} T_{b]}, \quad\left[G_{a}, G_{b}\right]=-S_{a b}, \\
{\left[S_{a b}, G_{c}\right] } & =2 \delta_{c[a} B_{b]}, \quad\left[J_{a b}, J_{c d}\right]=4 \delta_{[a[d} J_{c] b]}, \\
{\left[J_{a b}, X_{c}\right] } & =2 \delta_{c[a} X_{b]}, \quad\left[J_{a b}, S_{c d}\right]=4 \delta_{[a[d} S_{c] b]},
\end{aligned}
$$

where $X_{a} \in\left\{P_{a}, T_{a}, G_{a}, B_{a}\right\}$. The first line differs from the Bargmann algebra because $N$ is not central. Interestingly, this algebra can be obtained from a contraction of the direct sum of the Poincaré and Euclidean algebras in $d+1$ dimensions and underlies Newtonian gravity in the same way that the Poincare algebra underlies GR.

Off shell Newtonian gravity.-We now construct a Lagrangian depending on $\tau_{\mu}, h_{\mu \nu}, m_{\mu}, \Phi_{\mu \nu}$ that is invariant under the above gauge transformations. The unique twoderivative result is

$$
\begin{aligned}
\mathcal{L}= & -\frac{1}{16 \pi G} e\left[\hat{v}^{\mu} \hat{v}^{\nu} \bar{R}_{\mu \nu}-\tilde{\Phi} h^{\mu \nu} \bar{R}_{\mu \nu}\right. \\
& -\Phi_{\mu \nu} h^{\mu \rho} h^{\nu \sigma}\left(\bar{R}_{\rho \sigma}-a_{\rho} a_{\sigma}-\bar{\nabla}_{\rho} a_{\sigma}\right) \\
& \left.+\frac{1}{2} \Phi_{\mu \nu} h^{\mu \nu}\left[h^{\rho \sigma} \bar{R}_{\rho \sigma}-2 e^{-1} \partial_{\rho}\left(e h^{\rho \sigma} a_{\sigma}\right)\right]\right]
\end{aligned}
$$

where $e$ is the integration measure and we have omitted a possible cosmological constant term $e \Lambda$. The Lagrangian is obtained by starting with the (necessary) kinetic term $\hat{v}^{\mu} \hat{v}^{\nu} \bar{R}_{\mu \nu}$ and subsequently adding terms such that the entire expression is invariant under the torsional $\mathrm{U}(1)$ transformation as well the $\zeta_{\mu}$ transformation (10). This invariance follows from the Bianchi identities 


$$
\begin{aligned}
0= & e^{-1} \partial_{\rho}\left(e\left[h^{\rho \nu} \hat{v}^{\mu} \bar{R}_{\mu \nu}-\frac{1}{2} \hat{v}^{\rho} h^{\mu \nu} \bar{R}_{\mu \nu}\right]\right) \\
& +h^{\mu \rho} h^{\nu \sigma} \bar{K}_{\rho \sigma} \bar{R}_{\mu \nu}-\frac{1}{2} h^{\rho \sigma} \bar{K}_{\rho \sigma} h^{\mu \nu} \bar{R}_{\mu \nu} \\
0 & =h^{\mu \nu} h^{\rho \sigma} \bar{\nabla}_{\mu} \bar{R}_{\nu \rho}-\frac{1}{2} h^{\mu \sigma} h^{\nu \rho} \bar{\nabla}_{\mu} \bar{R}_{\nu \rho}
\end{aligned}
$$

which can be derived from $\bar{\nabla}_{[\lambda} \bar{R}_{\mu \nu] \sigma}^{\kappa}=0$.

Since we work with off shell TTNC geometries, we need to add the Lagrange multiplier term $\mathcal{L}_{\mathrm{LM}}=e \zeta^{\mu \nu}\left(\partial_{\mu} \tau_{\nu}-\right.$ $\left.\partial_{\nu} \tau_{\mu}\right)$ to the Lagrangian, where $\zeta^{\mu \nu}=-\zeta^{\nu \mu}$ obeying $\tau_{\mu}{ }^{\mu \nu}=0$, so that it only imposes $\tau \wedge d \tau=0$ but not $d \tau=0$ [23]. If we were to drop the condition $\tau_{\mu} \zeta^{\mu \nu}=0$ so that $\mathcal{L}_{\mathrm{LM}}$ enforces $d \tau=0$, the field $\zeta^{\mu \nu}$ would not decouple from the equations of motion. This is what happens in the 3D Chern-Simons actions for extended Bargmann algebras $[9,10]$, where $\zeta^{\mu \nu}=\epsilon^{\mu \nu \rho} \zeta_{\rho}$ with $\zeta_{\rho}$ associated with the central extension of the 3D Bargmann algebra.

We are going to compute the equations of motion by varying $\tilde{\Phi}, \hat{v}^{\mu}, \Phi_{\mu \nu}$, and $h^{\mu \nu}$. Let us define $\delta \mathcal{L}=-\frac{e}{8 \pi G}\left(E_{\tilde{\Phi}} \delta \tilde{\Phi}-E_{\mu} \delta \hat{v}^{\mu}+\frac{1}{2} E_{\mu \nu}^{h} \delta h^{\mu \nu}+\frac{1}{2} E^{\mu \nu} \delta \Phi_{\mu \nu}\right)$

where $E_{\tilde{\Phi}}=-\frac{1}{2} h^{\mu \nu} \bar{R}_{\mu \nu}$ and

$$
\begin{aligned}
E^{\mu \nu}= & -h^{\mu \rho} h^{\nu \sigma}\left(\bar{R}_{\rho \sigma}-a_{\rho} a_{\sigma}-\bar{\nabla}_{\rho} a_{\sigma}\right) \\
& +\frac{1}{2} h^{\mu \nu}\left[h^{\rho \sigma} \bar{R}_{\rho \sigma}-2 e^{-1} \partial_{\rho}\left(e h^{\rho \sigma} a_{\sigma}\right)\right] .
\end{aligned}
$$

The variations with respect to $P_{\mu}^{\rho} \delta \hat{v}^{\mu}$ with $P_{\mu}^{\rho}$ the spatial projector $P_{\mu}^{\rho} \equiv \delta_{\mu}^{\rho}+\hat{v}^{\rho} \tau_{\mu}$ gives

$$
h^{\rho \mu} E_{\mu}=-h^{\rho \mu} \hat{v}^{\nu} \bar{R}_{\mu \nu} .
$$

The remaining variations are $\tau_{\mu} \delta \hat{v}^{\mu}$ and $P_{\mu}^{\alpha} P_{\nu}^{\beta} \delta h^{\mu \nu}$. Defining $E_{h}^{\alpha \beta} \equiv h^{\mu \alpha} h^{\nu \beta} E_{\mu \nu}^{h}$, we find

$$
\begin{aligned}
-2 \hat{v}^{\mu} E_{\mu}= & -2 \tilde{\Phi} E_{\tilde{\Phi}}-\Phi_{\mu \nu} E^{\mu \nu}-h^{\mu \nu} \Phi_{\mu \nu} e^{-1} \partial_{\rho}\left(e h^{\rho \sigma} a_{\sigma}\right)+h^{\mu \rho} h^{\nu \sigma} \Phi_{\mu \nu}\left(\bar{\nabla}_{\rho} a_{\sigma}+a_{\rho} a_{\sigma}\right)+\left(h^{\rho \sigma} \bar{K}_{\rho \sigma}\right)^{2} \\
& \quad-h^{\rho \sigma} h^{\kappa \lambda} \bar{K}_{\rho \kappa} \bar{K}_{\sigma \lambda}+\bar{\nabla}_{\mu}\left[h^{\mu \rho} h^{\nu \sigma}\left(\bar{\nabla}_{\rho} \Phi_{\nu \sigma}-\bar{\nabla}_{\nu} \Phi_{\rho \sigma}\right)\right], \\
E_{h}^{\alpha \beta}= & \left(h^{\mu \alpha} h^{\nu \beta} \Phi_{\mu \nu}-\frac{1}{2} h^{\alpha \beta} h^{\mu \nu} \Phi_{\mu \nu}\right)\left[e^{-1} \partial_{\rho}\left(e h^{\rho \sigma} a_{\sigma}\right)+E_{\tilde{\Phi}}\right]-\frac{1}{2} h^{\alpha \beta} \Phi_{\mu \nu} E^{\mu \nu}+h^{\mu \alpha} \Phi_{\mu \rho} E^{\rho \beta}+h^{\mu \beta} \Phi_{\mu \rho} E^{\rho \alpha} \\
& -\frac{1}{2} h^{\rho \sigma} \Phi_{\rho \sigma} E^{\alpha \beta}+\tilde{\Phi} E^{\alpha \beta}-\frac{1}{2} h^{\alpha \beta}\left[\left(h^{\mu \nu} \bar{K}_{\mu \nu}\right)^{2}-h^{\mu \rho} h^{\nu \sigma} \bar{K}_{\mu \nu} \bar{K}_{\rho \sigma}\right]+\bar{\nabla}_{\rho}\left[\hat{v}^{\rho} h^{\mu \alpha} h^{\nu \beta} \bar{K}_{\mu \nu}-\hat{v}^{\rho} h^{\alpha \beta} h^{\mu \nu} \bar{K}_{\mu \nu}\right] \\
& +h^{\mu \alpha} h^{\nu \beta} \bar{\nabla}_{\mu} \partial_{\nu} \tilde{\Phi}+h^{\mu \alpha} h^{\nu \beta}\left(a_{\mu} \partial_{\nu} \tilde{\Phi}+a_{\nu} \partial_{\mu} \tilde{\Phi}\right)-h^{\alpha \beta} h^{\mu \nu} \bar{\nabla}_{\mu} \partial_{\nu} \tilde{\Phi}-2 h^{\alpha \beta} h^{\mu \nu} a_{\mu} \partial_{\nu} \tilde{\Phi} \\
& -\frac{1}{2} h^{\alpha \beta} h^{\mu \nu} h^{\rho \sigma}\left(\bar{\nabla}_{\mu}+a_{\mu}\right)\left(\bar{\nabla}_{\rho}+a_{\rho}\right) \Phi_{\nu \sigma}+h^{\mu \alpha} h^{\nu \beta} h^{\rho \sigma}\left(\bar{\nabla}_{\rho}+a_{\rho}\right)\left(\bar{\nabla}_{(\mu} \Phi_{\nu) \sigma}-\frac{1}{2} \bar{\nabla}_{\sigma} \Phi_{\mu \nu}\right) \\
& +\frac{1}{2} h^{\alpha \beta} h^{\mu \nu} h^{\rho \sigma}\left(\bar{\nabla}_{\mu}+a_{\mu}\right) \bar{\nabla}_{\nu} \Phi_{\rho \sigma}-\frac{1}{2} h^{\mu \alpha} h^{\nu \beta} h^{\rho \sigma} \bar{\nabla}_{\mu} \bar{\nabla}_{\nu} \Phi_{\rho \sigma} .
\end{aligned}
$$

We only need to consider the variation $P_{\mu}^{\alpha} P_{\nu}^{\beta} \delta h^{\mu \nu}$ because we are only interested in the spatial projection of $\Phi_{\mu \nu}$. By taking the trace of $E_{h}^{\alpha \beta}$ and using $\hat{v}^{\mu} E_{\mu}$, we find

$$
h^{\mu \nu} E_{\mu \nu}^{h}=-(d-2) \hat{v}^{\mu} E_{\mu}+\Phi_{\mu \nu} E^{\mu \nu}-(d-1)\left\{\hat{v}^{\mu} \hat{v}^{\nu} \bar{R}_{\mu \nu}-\left(\bar{\nabla}_{\mu}+a_{\mu}\right)\left[h^{\mu \nu} a_{\nu}\left(\tilde{\Phi}+\frac{1}{2} h^{\rho \sigma} \Phi_{\rho \sigma}\right)-h^{\mu \nu} h^{\rho \sigma} a_{\rho} \Phi_{\nu \sigma}\right]\right\},
$$

where we used the identity

$$
\hat{v}^{\mu} \hat{v}^{\nu} \bar{R}_{\mu \nu}=\left(h^{\mu \nu} \bar{K}_{\mu \nu}\right)^{2}-h^{\mu \rho} h^{\nu \sigma} \bar{K}_{\mu \nu} \bar{K}_{\rho \sigma}+3 h^{\mu \nu} a_{\mu} \partial_{\nu} \tilde{\Phi}+\bar{\nabla}_{\mu}\left(\hat{v}^{\mu} h^{\nu \rho} \bar{K}_{\nu \rho}+h^{\mu \nu} \partial_{\nu} \tilde{\Phi}\right)+2 \tilde{\Phi} e^{-1} \partial_{\mu}\left(e h^{\mu \nu} a_{\nu}\right) .
$$

Note that for $d \tau=0$ the field $\Phi_{\mu \nu}$ decouples.

It can be shown that these equations agree with [11], where they were obtained by expanding the Einstein equations in $1 / c^{2}$. However, [11] did not determine the equations of motion for $h^{\alpha \mu} h^{\beta \nu} \Phi_{\mu \nu}$, which we obtain by varying $\hat{v}^{\mu}$ and $h^{\mu \nu}$. These equations are essential in order to obtain a closed system of equations for the general case $d \tau \neq 0$. Importantly, we note that our action allows for geometries with strong gravitational fields and, in particular, those with $\tau$ not closed allow for nonrelativistic gravitational time dilation.

Given the gravity action with type II TNC gauge invariance, we need to understand how matter couples to such a geometry. This will be discussed in [22], but as 
remarked before this coupling will be markedly different than the known couplings of matter to type I TNC geometry [18,24-28]. One of the reasons is that, while in type I TNC geometry $m_{\mu}$ couples to the mass current, in type II it couples to the energy current. This will be further discussed in [22] by carefully studying the $1 / c$ limit of the worldline action of a relativistic particle as well as the known couplings of Poincaré invariant field theories to pseudoRiemannian geometry.

Here we will consider only the very special case of a static particle in order to obtain the Poisson equation from an action principle. A static point mass with mass density $\rho$ has a Lagrangian that is simply $\mathcal{L}_{m}=\alpha e \rho$ with $\alpha=$ $-[(d-2) / 2]$. Taking the trace of $E^{\mu \nu}$ gives $\bar{h}_{\mu \nu} E^{\mu \nu}+$ $(d-2) E_{\tilde{\Phi}}=-(d-1) e^{-1} \partial_{\rho}\left(e h^{\rho \sigma} a_{\sigma}\right)$. Varying $\mathcal{L}+\mathcal{L}_{m}$ tells us that the left-hand side vanishes and hence that $\partial_{\mu}\left(e h^{\mu \nu} a_{\nu}\right)=0$. Since $\tau \wedge d \tau=0$ we have that $h^{\mu \rho} h^{\nu \sigma}\left(\partial_{\rho} a_{\sigma}-\partial_{\sigma} a_{\rho}\right)=0$, so that $h^{\mu \nu} a_{\nu}=h^{\mu \nu} \partial_{\nu} F$ for some function $F$. Hence $\partial_{\mu}\left(e h^{\mu \nu} a_{\nu}\right)=0$ states that $F$ is a harmonic function on the $d$-dimensional Riemannian geometry of the hypersurface to which $\tau$ is orthogonal. Regularity requires $F$ to be constant and hence that $d \tau=0$, as desired in Newtonian gravity which has absolute time. What survives from (20) is then the equation $(d-1) \hat{v}^{\mu} \hat{v}^{\nu} \bar{R}_{\mu \nu}=-(d-2) \hat{v}^{\mu} E_{\mu}-h^{\mu \nu} E_{\mu \nu}^{h}$. Then, taking into account the matter contribution to $E_{\mu}$ and $E_{\mu \nu}^{h}$ in this equation, for which we use the variation $\delta \mathcal{L}_{m}=\alpha e \rho\left(\tau_{\mu} \delta \hat{v}^{\mu}-\right.$ $\left.\frac{1}{2} h_{\mu \nu} \delta h^{\mu \nu}\right)$, it follows that the equations of motion of $\mathcal{L}+\mathcal{L}_{m}$ with $d \tau=0$ are nothing else but Newton's law (6).

Discussion.-Among the numerous avenues that one may pursue following our action and corresponding novel geometry, we mention a few. It would be interesting to (i) examine if there exists a geometric construction that gives type II TNC geometry from some Lorentzian starting point, just like type I follows from null reduction of a Lorentzian metric, (ii) perform a Hamiltonian analysis along with determining the asymptotic symmetries and examining the solution space of the theory, and (iii) work out how particles, strings, and branes probe type II TNC geometry and see if the equations of motion of the nonrelativistic gravity action can be related to consistency conditions of some type of string theory (see [29-31] for nonrelativistic strings in the context of type I TNC geometry). Finally, there are undoubtedly also exciting applications in the realm of the AdS/CFT correspondence and generalizations thereof.

We thank Eric Bergshoeff, Dieter van den Bleeken, Shira Chapman, Lorenzo Di Pietro, José Figueroa-O'Farrill, Gerben Oling, Manus Visser, and Ziqi Yan for useful discussions. We also thank José Figueroa-O'Farrill for pointing out that the algebra (11) can be obtained from a contraction. The work of D. H. is supported by the Swiss National Science Foundation through the NCCR SwissMAP. The work of J. H. is supported by the Royal Society University Research Fellowship "Non-Lorentzian
Geometry in Holography" (Grant No. UF160197). The work of N. O. is supported in part by the project "Towards a deeper understanding of black holes with non-relativistic holography" of the Independent Research Fund Denmark (Grant No. DFF-6108-00340). J. H. and N. O. thank the Perimeter Institute for hospitality during completion of this work.

\section{*dehansen@phys.ethz.ch}

†Jelle.Hartong@ed.ac.uk

obers@nbi.ku.dk

[1] E. Cartan, Sur les variétés à connexion affine et la théorie de la rélativité généralisée (première partie), Ann. Éc. Norm. Super. 40, 325 (1923).

[2] E. Cartan, Sur les variétés à connexion affine et la théorie de la rélativité généralisée (première partie) (suite), Ann. Éc. Norm. Super. 41, 1 (1924).

[3] A. Trautman, Sur la theorie newtonienne de la gravitation, C.R. Acad. Sci. (Paris) 247, 617 (1963).

[4] P. Havas, Four-dimensional formulations of newtonian mechanics and their relation to the special and the general theory of relativity, Rev. Mod. Phys. 36, 938 (1964).

[5] M. H. Christensen, J. Hartong, N. A. Obers, and B. Rollier, Boundary stress-energy tensor and Newton-Cartan geometry in Lifshitz holography, J. High Energy Phys. 01 (2014) 057.

[6] M. H. Christensen, J. Hartong, N. A. Obers, and B. Rollier, Torsional Newton-Cartan geometry and Lifshitz holography, Phys. Rev. D 89, 061901 (2014).

[7] J. Hartong and N. A. Obers, Hořava-Lifshitz gravity from dynamical Newton-Cartan geometry, J. High Energy Phys. 07 (2015) 155.

[8] H. R. Afshar, E. A. Bergshoeff, A. Mehra, P. Parekh, and B. Rollier, A Schrödinger approach to Newton-Cartan and Hořava-Lifshitz gravities, J. High Energy Phys. 04 (2016) 145.

[9] E. A. Bergshoeff and J. Rosseel, Three-Dimensional Extended Bargmann Supergravity, Phys. Rev. Lett. 116, 251601 (2016).

[10] J. Hartong, Y. Lei, and N. A. Obers, Nonrelativistic ChernSimons theories and three-dimensional Hořava-Lifshitz gravity, Phys. Rev. D 94, 065027 (2016).

[11] D. Van den Bleeken, Torsional Newton-Cartan gravity from the large c expansion of general relativity, Classical Quantum Gravity 34, 185004 (2017).

[12] G. Dautcourt, Post-Newtonian extension of the NewtonCartan theory, Classical Quantum Gravity 14, A109 (1997).

[13] W. Tichy and E. E. Flanagan, Covariant formulation of the post-1-Newtonian approximation to general relativity, Phys. Rev. D 84, 044038 (2011).

[14] R. Andringa, E. Bergshoeff, S. Panda, and M. de Roo, Newtonian gravity and the Bargmann algebra, Classical Quantum Gravity 28, 105011 (2011).

[15] C. Duval and H. P. Kunzle, Minimal gravitational coupling in the Newtonian theory and the covariant Schrodinger equation, Gen. Relativ. Gravit. 16, 333 (1984).

[16] R. De Pietri, L. Lusanna, and M. Pauri, Standard and generalized Newtonian gravities as 'gauge' theories of the 
extended Galilei group. I. The standard theory, Classical Quantum Gravity 12, 219 (1995).

[17] X. Bekaert and K. Morand, Connections and dynamical trajectories in generalised Newton-Cartan gravity I. An intrinsic view, J. Math. Phys. (N.Y.) 57, 022507 (2016).

[18] K. Jensen, On the coupling of Galilean-invariant field theories to curved spacetime, SciPost Phys. 5, 011 (2018).

[19] J. Hartong, E. Kiritsis, and N. A. Obers, Lifshitz spacetimes for Schrödinger holography, Phys. Lett. B 746, 318 (2015).

[20] G. Festuccia, D. Hansen, J. Hartong, and N. A. Obers, Torsional Newton-Cartan geometry from the Noether procedure, Phys. Rev. D 94, 105023 (2016).

[21] B. Julia and H. Nicolai, Null killing vector dimensional reduction and Galilean geometrodynamics, Nucl. Phys. B439, 291 (1995).

[22] D. Hansen, J. Hartong, and N. A. Obers (to be published).

[23] The field $\zeta^{\mu \nu}$ can be written as $\zeta^{\mu \nu}=h^{\mu \rho} h^{\nu \sigma} \zeta_{\rho \sigma}$, where $\zeta_{\rho \sigma}$ is a Lagrange multiplier for the TTNC condition. It can be shown that the variations with respect to $\hat{v}^{\mu}$ and $P_{\rho}^{\mu} P_{\sigma}^{\nu} \delta h^{\rho \sigma}$ [as explained around Eq. (20) this is the relevant variation to consider] do not contribute to the equations of motion due to the TTNC condition. Therefore, the field $\zeta^{\mu \nu}$ decouples from the dynamics.
[24] J. Hartong, E. Kiritsis, and N. A. Obers, Schrödinger invariance from Lifshitz isometries in holography and field theory, Phys. Rev. D 92, 066003 (2015).

[25] M. Geracie, K. Prabhu, and M. M. Roberts, Fields and fluids on curved non-relativistic spacetimes, J. High Energy Phys. 08 (2015) 042.

[26] J. F. Fuini, A. Karch, and C. F. Uhlemann, Spinor fields in general Newton-Cartan backgrounds, Phys. Rev. D 92 , 125036 (2015).

[27] E. Bergshoeff, J. Rosseel, and T. Zojer, Non-relativistic fields from arbitrary contracting backgrounds, Classical Quantum Gravity 33, 175010 (2016).

[28] G. Festuccia, D. Hansen, J. Hartong, and N. A. Obers, Symmetries and couplings of non-relativistic electrodynamics, J. High Energy Phys. 11 (2016) 037.

[29] T. Harmark, J. Hartong, and N. A. Obers, Nonrelativistic strings and limits of the AdS/CFT correspondence, Phys. Rev. D 96, 086019 (2017).

[30] E. Bergshoeff, J. Gomis, and Z. Yan, Nonrelativistic string theory and T-duality, J. High Energy Phys. 11 (2018) 133.

[31] T. Harmark, J. Hartong, L. Menculini, N. A. Obers, and Z. Yan, Strings with non-relativistic conformal symmetry and limits of the AdS/CFT correspondence, J. High Energy Phys. 11 (2018) 190. 